\title{
Hybrid origin of a B chromosome (PSR) in the parasitic wasp Nasonia vitripennis
}

\author{
Bryant F. McAllister*, John H. Werren \\ Department of Biology, University of Rochester, Rochester, NY 14627, USA
}

Received: 19 December 1996; in revised form: 14 April 1997 / Accepted: 24 April 1997

\begin{abstract}
Little is known about the origin and evolution of supernumerary (B) chromosomes. This study utilizes molecular markers to examine the evolutionary history and microstructural organization of the supernumerary paternal-sex-ratio (PSR) chromosome of the parasitic wasp Nasonia vitripennis. Copies of the retrotransposon NATE were previously isolated from PSR and the genomes of $N$. vitripennis and related wasp species. A phylogenetic analysis of sequences representing 29 elements from PSR and seven wasp species, coupled with a hybridization analysis of elements in genomic DNA provides evidence that PSR was recently transferred into $N$. vitripennis from a species in the genus Trichomalopsis. A linear region of the PSR chromosome was compared by Southern blot analysis with genomic DNA from $N$. vitripennis, Nasonia longicornis, Trichomalopsis americanus, and Trichomalopsis dubius. A region organized similarly to the region on PSR was not evident in any of the species, thus a progenitor region was not identified. However, the hybridizations revealed that this region of PSR is primarily composed of repetitive sequences that appear dispersed in these wasp genomes, and might represent additional mobile elements. At least three different dispersed repeats are present in the $18 \mathrm{~kb}$ region of PSR. The abundance of tandem and dispersed repetitive sequences in this relatively small region provides additional evidence for the degenerate structure of the PSR chromosome.
\end{abstract}

\footnotetext{
Edited by: P.B. Moens

*Present address: (before August, 1997) Department of Ecology and Evolution, The University of Chicago, 1101 E. 57th St., Chicago, IL 60637-1573, USA, (e-mail: bryant@pondside.uchicago.edu); (after August, 1997) ICAPB, University of Edinburgh, Ashworth Laboratories, West Mains Road, Edinburgh EH9 3JT, UK
}

Correspondence to: B.F. McAllister

\section{Introduction}

Supernumerary (or B) chromosomes ${ }^{1}$ have been described in all major groups of animals and plants (Jones and Rees 1982; Beukeboom 1994), and are fundamentally similar to the accessory chromosomes or plasmids that are found in lower eukaryotes and bacteria. The critical characteristic of supernumerary elements is that they are unnecessary for survival of the organism in which they occur. As the definition by Camacho and Parker explicitly states, the B chromosome "follows its own evolutionary pathway" (Beukeboom 1994). Because of this autonomy from the standard (or A) chromosomal complement, B chromosomes are excellent systems for studying evolutionary dynamics. Studies on the evolutionary dynamics of Bs have primarily concentrated on examining the maintenance of the chromosomes within populations (Jones 1991). Very little is known about other evolutionary aspects, such as their origins, processes of molecular evolution, and ultimate evolutionary fates.

Identifying the origins of $\mathrm{B}$ chromosomes has been a lingering problem. Two primary sources for B chromosomes have been considered (Jones and Rees 1982; Green 1990); either an intragenomic fragment acquires the characteristics of a $\mathrm{B}$, or interspecific hybridization provides foreign DNA that evolves into a B chromosome. Both of these scenarios share the central feature that a DNA fragment released from selective constraints evolves toward independence as a B chromosome. The first scenario postulates that B chromosomes arise from duplicated or fragmented pieces within the genome. Apparent examples occur in the plant Crepis capillaris (Jamilena et al. 1994a, b, 1995) and the fly Drosophila subsilvestris (Gutknecht et al. 1995). In both cases, molecular analyses indicate that repetitive sequences on the Bs are shared with the A chromosomes, but progenitor

\footnotetext{
${ }^{1} \mathrm{~B}$ chromosome, "a dispensable supernumerary chromosome that does not recombine with the A chromosomes and follows its own evolutionary pathway". (J.P.M. Camacho and J.S. Parker, Beukeboom 1994)
} 
regions have not been identified and direct sequence comparisons have not been performed. Another pathway for the evolution of a $\mathrm{B}$ from within the genome is the acquisition of $\mathrm{B}$ characteristics by sex-limited chromosomes (W or Y), as has been postulated for the New Zealand frog Leiopelma hochstetteri (Green et al. 1993; T.F. Sharbel, personal communication). The other proposed source for the origin of B chromosomes is foreign DNA introduced from a closely related species through hybridization. This scenario has been reproduced experimentally with two plant species, where chromosomes from Coix gigantea were incorporated as supernumeraries in plants with an intact Coix aquatica chromosomal complement (Sapre and Deshpande 1987). Also, molecular data are suggestive of an interspecific origin of the $\mathrm{B}$ in the Australian annual Brachycome dichromosomatica (John et al. 1991; Leach et al. 1995). A sequence isolated from the B chromosome cross-hybridized to genomic DNA from other Brachycome species, but not to the A chromosomes of $B$. dichromosomatica. Another possible example of $\mathrm{B}$ chromosome origin following interspecific hybridization is in the gynogenetic Amazon molly Poeccilia formosa (Schartl et al. 1995; McVean 1995).

Comparisons of DNA similarity are necessary for determining $\mathrm{B}$ chromosome origins, although several factors may dictate the pattern of similarity. Both the mechanism of origin and the age of the $\mathrm{B}$ chromosome will influence the composition of a $\mathrm{B}$ relative to the A chromosomal complement. Recent intragenomic or hybrid origins initially create different relationships between the $\mathrm{B}$ and resident chromosomal complement, but over time the initial pattern will be obscured. If a B chromosome arises from within the genome, initially it will be very similar to the progenitor region. Through time the $\mathrm{B}$ will diverge quantitatively through sequence evolution and qualitatively through rearrangements. Selection pressure to reduce pairing with the progenitor region during meiosis, which would cause genetic imbalance, may drive sequence divergence from the progenitor region in the A complement (Amos and Dover 1981). Even without the selective pressure to reduce B/A pairing, the absence of recombination and selection presumably dictates the loss of functional loci on B chromosomes (Green 1990). Under a hybrid origin, the B is originally dissimilar from the A complement. Over time, the $\mathrm{B}$ exchanges mobile elements with the new resident genome, thus becoming qualitatively more similar in repetitive DNA content. Although there are possible examples of both intragenomic and intergenomic origins for particular B chromosomes, none of the systems have been examined to the extent where the mechanism of origin and pattern of molecular evolution can be clearly documented.

Paternal sex ratio (PSR) is a $\mathrm{B}$ chromosome in the parasitic wasp Nasonia vitripennis (Nur et al. 1988; Werren 1991). The transmission pattern of this chromosome is an extreme example of the independent nature of B chromosomes. Only male wasps carry PSR and the chromosome is transmitted by sperm (Werren and van den Assem 1986; Beukeboom and Werren 1993a). Fol- lowing fertilization with a PSR-bearing sperm, the maternal chromosomes and PSR undergo the first mitotic division in the zygote, whereas the standard paternal chromosomes form a chromatin mass that fails to divide (Werren et al. 1987; Reed and Werren 1995). Loss of the paternal chromosomes results in the transformation of a diploid zygote into a haploid zygote that develops into a male, owing to haplodiploid sex determination. Molecular analyses of PSR have characterized a number of repetitive sequences from this chromosome (Nur et al. 1988; Eickbush et al. 1992; McAllister 1995). Three different families of PSR-specific tandemly repeated sequences (PSR2, PSR18, PSR22), a tandem repeat (PSR79) that is also present in the A complement, and a retrotransposable element (NATE) have all been cloned and characterized from PSR. These isolated sequences indicate that the overall composition of PSR is distinct from the A chromosomes of $N$. vitripennis. Cytological1y, PSR is also heterochromatic (Reed 1993).

In previous papers (Nur et al. 1988; Werren 1991; McAllister 1995), a hybrid origin of the PSR chromosome has been proposed. Based upon the geographic distribution of PSR (restricted to western North America in an area of sympatry with the sibling species Nasonia longicornis) and evidence that interspecific cytoplasmic incompatibility results in the generation of de novo chromosomal fragments (Ryan et al. 1985; Beukeboom and Werren 1993b), it was proposed that PSR arose as a chromosomal fragment from $N$. longicornis generated by interspecific hybridization with $N$. vitripennis. In this paper, a sequence analysis of the retrotransposon NATE is used to examine directly the evolutionary history of the PSR chromosome. Sequences of 29 elements isolated from PSR and the genomes of seven wasp species are analyzed. NATE appears useful for inferring the history of PSR, because the phylogenetic pattern exhibited by a larger sample of elements was consistent with vertical maintenance of the element in this group of wasps (McAllister and Werren 1997). Furthermore, in an attempt to identify the progenitor region from which PSR was derived, a linear region of PSR is used to examine the organization of cross-hybridizing sequences in the A complements of four wasp species. Results support a hybrid origin of PSR, but through hybridization between $N$. vitripennis and a wasp from the closely related genus Trichomalopsis.

\section{Materials and methods}

Genomic DNA. Nine species of parasitoid wasps were used in this study. These wasp species are $N$. vitripennis (with PSR and without PSR), N. longicornis, Nasonia giraulti, Trichomalopsis (=Eupteromalus) americanus, Trichomalopsis (=Eupteromalus) dubius, Urolepis rufipes, Muscidifurax uniraptor, Muscidifurax raptorellus, and Muscidifurax raptor. All of these species are classified in the tribe Pteromalini within the family Pteromalidae (Burks 1979). Wasps were cultured in the laboratory on Sarcophaga bullata or Musca domestica pupae. Total genomic DNA was extracted from pooled wasps of a single strain using standard techniques for phenol/chloroform extraction and ethanol precipitation as previously described (McAllister 1995; McAllister and Werren 1997). 
Phylogeny of NATE. The sequences of NATE used in this study were previously used in a more extensive analysis of element phylogeny (McAllister and Werren 1997), and the sequences are available in GenBank (U29470-U29473, U69494, U69496U69506, U69514-U69522, U69525-U69527, U69529). Procedures for isolating these sequences of NATE have been presented (McAllister 1995; McAllister and Werren 1997). Briefly, $\lambda$ clones containing copies of NATE on the PSR chromosome were isolated from a genomic library containing DNA from PSR males in the MI strain of $N$. vitripennis. Two cross-hybridizing elements were isolated from a genomic library of the IV-14 strain of $N$. longicornis. An approximately 900 bp region encompassing the reverse transcriptase (RT) domain was sequenced from four PSR and two $N$. longicornis elements. Regions of sequence conservation were identified and two sets of primers were designed for amplifying elements from genomic DNA using the polymerase chain reaction (PCR). The g-primer set (N6, 5' TAC ACC TCA AAG GCA CAG; N14, 5' AGA CCA GCT TCG TTT ATC C) was designed to amplify a wide range of elements. The p primers (N12, 5' GCC TCC CTC TCG TCT GCA; N13, 5' CAA TGG GTT TGA CTC GTT CG) form a primer set designed preferentially to amplify elements more closely related to those on PSR. A $700 \mathrm{bp}$ consensus sequence is shared by both reaction products, encompassing $240 \mathrm{bp}$ preceding and $460 \mathrm{bp}$ into the RT domain of the elements.

The previous study of 43 element sequences isolated from nine species of parasitoid wasps indicated that two major subfamilies (I and II) of NATE are present in these wasps (McAllister and Werren 1997). All of the elements isolated from PSR were contained in subfamily I, so this analysis concentrated on this subfamily. Sequences from 29 elements representing 7 species and PSR were analyzed. Elements were examined from PSR, $N$. longicornis, $N$. giraulti, T. americanus, $T$. dubius, $U$. rufipes, $M$. raptorellus, and $M$. uniraptor. Nomenclature of the element sequences is given by the species name, the method of obtaining the sequence ( $\mathrm{c}=\mathrm{clone}$ from genomic library, $\mathrm{p}=\mathrm{PCR}$ product using the $\mathrm{p}$-primer set, $\mathrm{g}=\mathrm{PCR}$ product using the g-primer set), and a number identifying the sequence.

The sequences were visually aligned to minimize the number of gaps. A parsimony analysis to determine the relationships among the sequences was performed using PAUP ver. 3.1 (Swofford 1991). The heuristic search option was used with the sequences added randomly 50 times. Three sequences from subfamily II were designated as a user-defined outgroup for rooting the subfamily I sequences. To determine the confidence in each node in the phylogenetic tree, 500 bootstrap replications were performed using the heuristic search option with ten random additions. Pair-wise differences were also calculated using PAUP.

Southern blotting of NATE. The genomic DNAs were screened for the presence of NATE using hybridization. Genomic DNA from each wasp species was digested with AluI following the manufacturer's recommendations (BRL). The quality and quantity of digested DNAs were visually compared by ethidium bromide staining following electrophoresis of a subset of the reactions. Equal amounts of digested DNAs were electrophoresed in a $1.5 \%$ agarose gel and transferred to a nylon membrane. An N6/N14 PCR product from a $\lambda$ clone $(\lambda \mathrm{P} 16)$ containing a PSR-inserted NATE (PSR c16) was labeled with $\left[\alpha^{32} \mathrm{P}\right] \mathrm{dATP}$ using random primer labeling following the manufacturer's (BRL) suggestions and used as a probe. The hybridization solution contained $5 \times$ SSC, $5 \times$ Denhardt's solution, $125 \mathrm{mg} / \mathrm{ml}$ denatured calf thymus DNA, and $1 \%$ SDS (Ausubel et al. 1992). Hybridization was performed for $16 \mathrm{~h}$ at $65^{\circ} \mathrm{C}$. The final wash of the membrane was at $65^{\circ} \mathrm{C}$ with a solution of $0.1 \times \mathrm{SSC}$ and $0.1 \%$ SDS. $(1 \times \mathrm{SSC}$ is $0.15 \mathrm{M} \mathrm{NaCl}, 0.015 \mathrm{M}$ sodium citrate.)

Structure of PSR. The structure of an $18 \mathrm{~kb}$ cloned region from the PSR chromosome was examined. This region $(\lambda P 17)$ of the PSR chromosome was isolated in a previous study (McAllister 1995), because it contains a partial copy of NATE. In addition to NATE (PSR c17), which was truncated by the cloning site at one end of the insert, the clone contains $11 \mathrm{~kb}$ flanking the intact end of this element. Subclones from $\lambda \mathrm{P} 17$ were obtained in two rounds of double-digestion and ligating into Bluescript (Stratagene), one with EcoRI and HindIII and the other with EcoRI and HincII. A restriction map of this region was obtained by hybridizing a blot of fully and partially digested $\lambda$ P17 DNA with specific subclones from the insert.

Four subclones from the $\lambda \mathrm{P} 17$ region were used to examine the structure of cross-hybridizing sequences on PSR and in total genomic DNA of $N$. vitripennis (without PSR), $N$. longicornis, $T$. americanus, and T. dubius. Genomic DNA from each strain and $\lambda$ P17 was digested with HindIII; the samples were divided into four aliquots containing equal amounts of DNA and separated in an $0.8 \%$ agarose gel. After the DNAs had been transferred to a nylon membrane, the membrane was cut into four pieces, each containing HindIII-digested DNA from all the wasp strains and $\lambda \mathrm{P} 17$. Inserts from subcloned regions of $\lambda \mathrm{P} 17$ were amplified from plasmids using the T3 and T7 primer sites located at both ends of the polycloning region in Bluescript. These PCR products were labeled by random primer labeling and used as probes. Labeling of probes and hybridization were carried out under the same conditions as above. Each of the four probes was hybridized to one of the four membranes. Final washes of the membranes were in a solution of $0.2 \times \mathrm{SSC}$ and $0.1 \%$ SDS at $65^{\circ} \mathrm{C}$. This procedure provided an independent hybridization for each probe to identical DNA samples, which were electrophoresed under the same conditions.

Partial crude sequence was obtained from the region of $\lambda \mathrm{P} 17$ flanking the copy of NATE. Approximately $300 \mathrm{bp}$ was read into each end of the plasmid subclones, and most of the sequence was obtained by reading only a single strand. Sequencing reactions were performed on chemically denatured $(\mathrm{NaOH})$ double-stranded plasmid DNA (Ausubel et al. 1992) using the T3 and T7 sequencing primers. Reactions were performed with the Sequenase kit (USB), labeling with $\left[\alpha^{35}\right.$ S $]$ dATP, and electrophoresing through buffer gradient gels. Manipulation of the sequence was performed using ESEE ver. 1.0 (Cabot and Beckenbach 1989) and GCG (Genetic Computer Group, Madison, Wis.). Homology searches of the sequence databases were performed with the BLASTN and BLASTX algorithms (Altschul et al. 1990; Gish and States 1993) using NCBI (National Center for Biotechnology Information) facilities accessed through the network server.

\section{Results}

\section{Phylogenetic analysis of NATE to determine PSR history}

This analysis of the relationships among these NATE sequences was performed to investigate the evolutionary history of PSR. The phylogenetic analysis revealed that elements on PSR form a very closely related group (Fig. 1). All but two of the PSR elements are contained in a monophyletic group that is supported by a relatively high (84\%) bootstrap value, considering there are only two diagnostic nucleotides supporting this group. This indicates that these elements have recently replicated from a common ancestral element. Although the close relationship among these elements apparently represents a recent diversification, the seven PSR elements exhibit a $1.1 \%$ mean total pair-wise difference throughout the 700 bp region (Table 1). Therefore, a substantial amount of sequence divergence has occurred among these elements. 


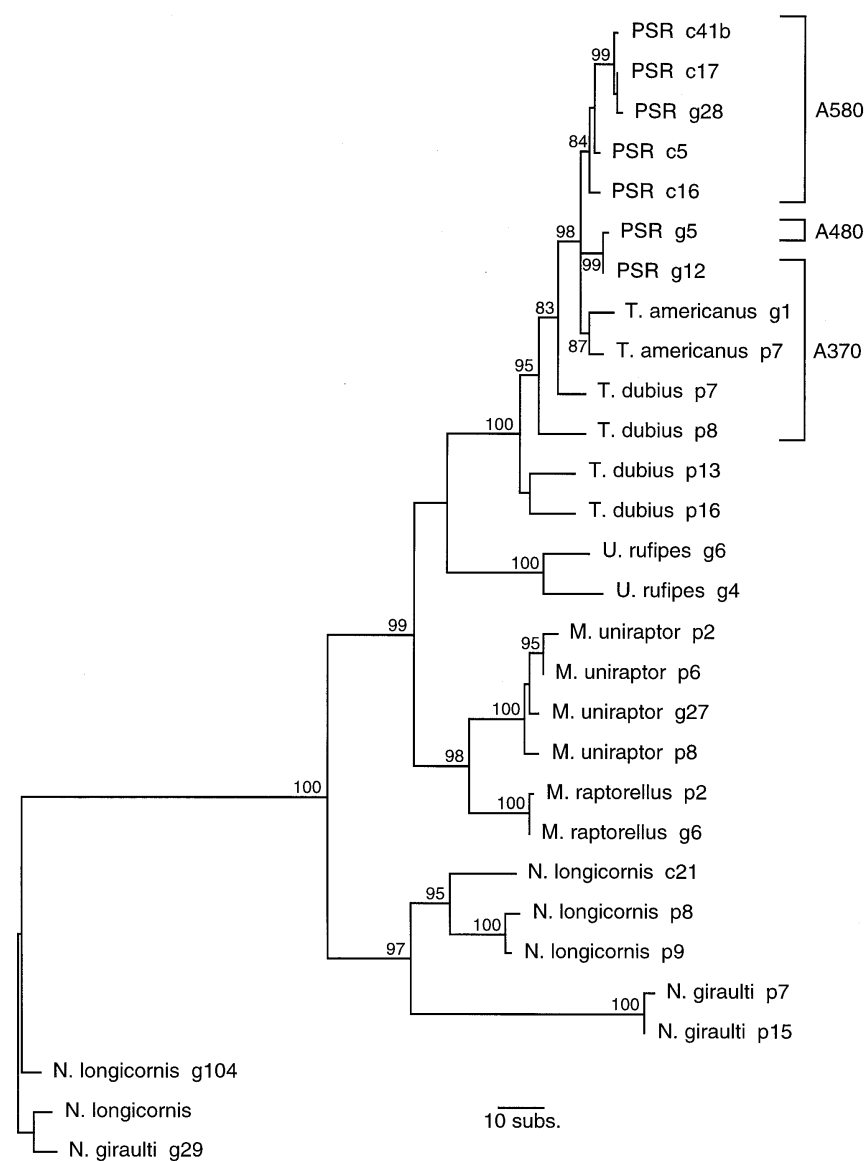

Fig. 1. Phylogenetic relationships among element sequences from the paternal-sex-ratio (PSR) chromosome of Nasonia vitripennis and seven wasp species. This is one of the three equally parsimonious trees obtained with a minimum length of 409 substitutions. Bootstrap values are presented for the nodes with greater than $80 \%$ support
Most of these elements designated as occurring on PSR have been independently verified as inserts on the chromosome. Four of the seven PSR elements were obtained in $\lambda$ clones (identified with "c" in Fig. 1) by screening a genomic library, and unique PCR and/or Southern blot assays were developed to detect the presence of the regions containing these elements (McAllister et al. in preparation). By screening genomic DNA extracted from a strain of $N$. vitripennis with and without PSR, these elements have been specifically localized to the chromosome. The three elements obtained by PCR were not independently verified as inserts on this chromosome, but similar elements were not obtained upon amplification from genomic DNA isolated from the same $N$. vitripennis strain (MI) without PSR, using the p-element primers.

As seen in Fig. 1, element sequences from the genome of $T$. americanus are grouped as the closest relatives to the PSR elements; furthermore, the elements on PSR are contained in a clade represented by elements obtained from both species of Trichomalopsis. This clade of PSR/Trichomalopsis elements is strongly supported by a bootstrap value of $100 \%$, indicating that this relationship is not due to chance. In contrast, elements from the Nasonia species were distantly related to the PSR elements (Fig. 1). Failure to amplify PSR-like elements in any of the three Nasonia species was not due to sampling error during cloning of the PCR products. Two different primer pairs were used to amplify elements from genomic DNA, and restriction digestion of these products and visualization in ethidium bromide-stained agarose gels revealed that PSR-like elements were not amplified from the genomes of any of the three Nasonia species. Therefore, NATE elements on PSR are more closely related to those found in Trichomalopsis than to those in Nasonia.

Table 1. Mean $( \pm \mathrm{SD})$ pair-wise sequence differences within and among element groups

\begin{tabular}{|c|c|c|c|c|c|c|c|c|c|c|}
\hline & PSR-7a & T. am.-2 & T. dub.-4 & U. ruf.-2 & M. uni.-4 & M. rel.-2 & N. lon.-3 & N. gir.-2 & N. lon. $-2^{b}$ & N. gir. $-1^{b}$ \\
\hline PSR-7 & $\begin{array}{l}0.011 \\
( \pm 0.007)\end{array}$ & $\begin{array}{l}0.018 \\
( \pm 0.003)\end{array}$ & $\begin{array}{l}0.034 \\
( \pm 0.008)\end{array}$ & $\begin{array}{l}0.091 \\
( \pm 0.002)\end{array}$ & $\begin{array}{l}0.092 \\
( \pm 0.003)\end{array}$ & $\begin{array}{l}0.091 \\
( \pm 0.002)\end{array}$ & $\begin{array}{l}0.115 \\
( \pm 0.003)\end{array}$ & $\begin{array}{l}0.161 \\
( \pm 0.003)\end{array}$ & $\begin{array}{l}0.141 \\
( \pm 0.002)\end{array}$ & $\begin{array}{l}0.147 \\
( \pm 0.002)\end{array}$ \\
\hline T. am.-2 & & $\begin{array}{l}0.012 \\
-\end{array}$ & $\begin{array}{l}0.036 \\
( \pm 0.008)\end{array}$ & $\begin{array}{l}0.092 \\
( \pm 0.002)\end{array}$ & $\begin{array}{l}0.093 \\
( \pm 0.002)\end{array}$ & $\begin{array}{l}0.094 \\
( \pm 0.002)\end{array}$ & $\begin{array}{l}0.117 \\
( \pm 0.003)\end{array}$ & $\begin{array}{l}0.165 \\
( \pm 0.004)\end{array}$ & $\begin{array}{l}0.146 \\
( \pm 0.002)\end{array}$ & $\begin{array}{l}0.152 \\
( \pm 0.002)\end{array}$ \\
\hline T. dub.-4 & & & $\begin{array}{l}0.033 \\
( \pm 0.004)\end{array}$ & $\begin{array}{l}0.085 \\
( \pm 0.002)\end{array}$ & $\begin{array}{l}0.084 \\
( \pm 0.005)\end{array}$ & $\begin{array}{l}0.083 \\
( \pm 0.005)\end{array}$ & $\begin{array}{l}0.111 \\
( \pm 0.002)\end{array}$ & $\begin{array}{l}0.156 \\
( \pm 0.002)\end{array}$ & $\begin{array}{l}0.145 \\
( \pm 0.006)\end{array}$ & $\begin{array}{l}0.152 \\
( \pm 0.006)\end{array}$ \\
\hline U. ruf.-2 & & & & $\begin{array}{l}0.003 \\
-\end{array}$ & $\begin{array}{l}0.094 \\
( \pm 0.002)\end{array}$ & $\begin{array}{l}0.091 \\
( \pm 0.001)\end{array}$ & $\begin{array}{l}0.116 \\
( \pm 0.006)\end{array}$ & $\begin{array}{l}0.158 \\
( \pm 0.005)\end{array}$ & $\begin{array}{l}0.146 \\
( \pm 0.003)\end{array}$ & $\begin{array}{l}0.154 \\
( \pm 0.006)\end{array}$ \\
\hline M. uni.-4 & & & & & $\begin{array}{l}0.009 \\
( \pm 0.003)\end{array}$ & $\begin{array}{l}0.042 \\
( \pm 0.003)\end{array}$ & $\begin{array}{l}0.115 \\
( \pm 0.002)\end{array}$ & $\begin{array}{l}0.156 \\
( \pm 0.003)\end{array}$ & $\begin{array}{l}0.154 \\
( \pm 0.003)\end{array}$ & $\begin{array}{l}0.161 \\
( \pm 0.003)\end{array}$ \\
\hline M. rel.-2 & & & & & & $\begin{array}{l}0.001 \\
-\end{array}$ & $\begin{array}{l}0.109 \\
( \pm 0.002)\end{array}$ & $\begin{array}{l}0.148 \\
( \pm 0.002)\end{array}$ & $\begin{array}{l}0.142 \\
( \pm 0.002)\end{array}$ & $\begin{array}{l}0.150 \\
( \pm 0.001)\end{array}$ \\
\hline N. lon.-3 & & & & & & & $\begin{array}{l}0.030 \\
( \pm 0.021)\end{array}$ & $\begin{array}{l}0.105 \\
( \pm 0.003)\end{array}$ & $\begin{array}{l}0.146 \\
( \pm 0.003)\end{array}$ & $\begin{array}{l}0.153 \\
( \pm 0.003)\end{array}$ \\
\hline N. gir.-2 & & & & & & & & $\begin{array}{l}0.003 \\
-\end{array}$ & $\begin{array}{l}0.174 \\
( \pm 0.002)\end{array}$ & $\begin{array}{l}0.180 \\
( \pm 0.002)\end{array}$ \\
\hline N. lon.-2 & & & & & & & & & $\begin{array}{l}0.016 \\
-\end{array}$ & $\begin{array}{l}0.015 \\
( \pm 0.003)\end{array}$ \\
\hline
\end{tabular}

T. am., Trichomalopsis americanus; T. dub., T. dubius; U. ruf., Urolepsis rufipes; M. uni., Muscidifurax uniraptor; M. rel., M. raptorellus; M. lon., Nasonia longicornis; N. gir., N. giraulti a Number of elements in group

b Outgroup elements 


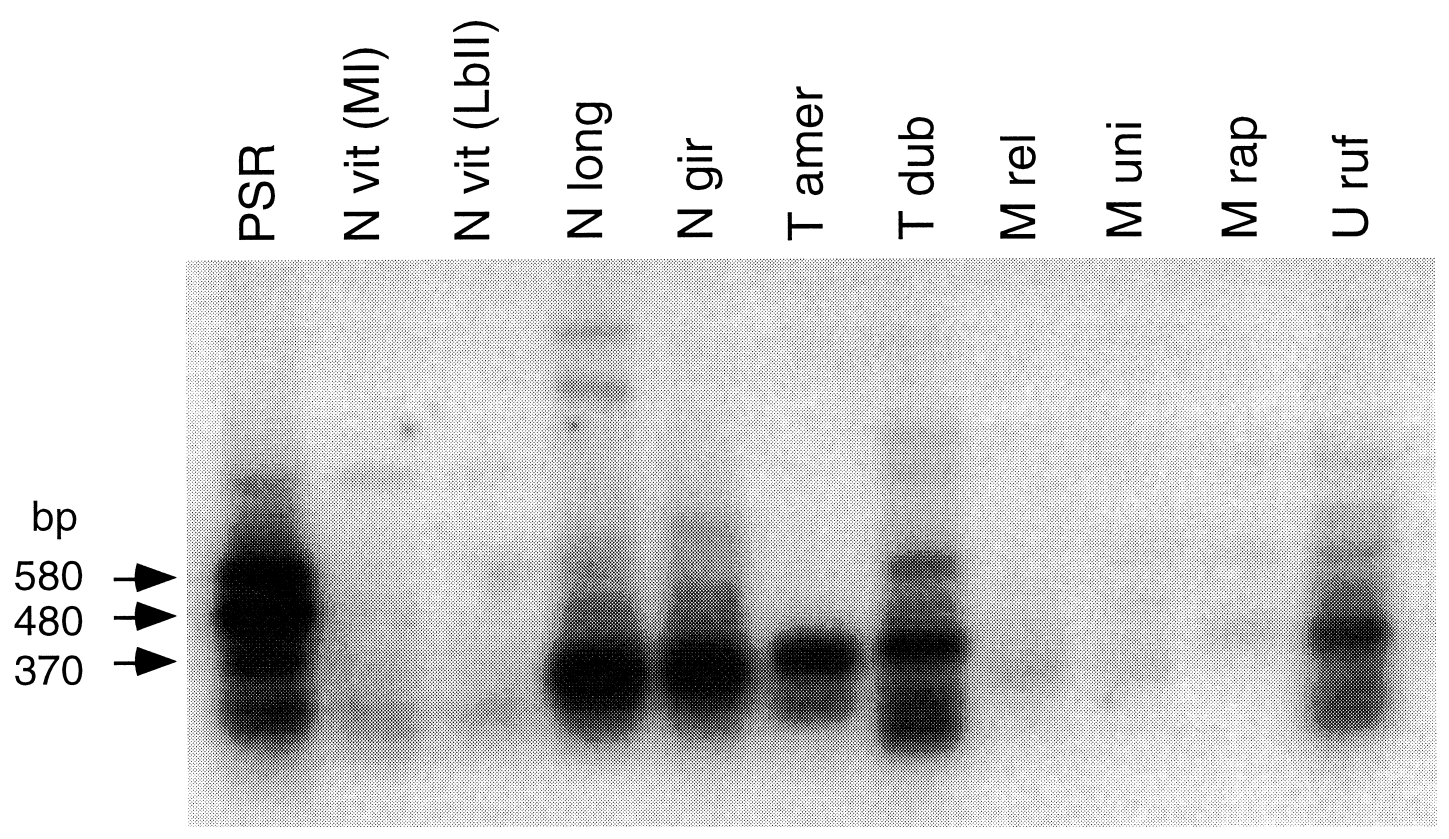

Fig. 2. Hybridization of the reverse transcriptase (RT) region of NATE to restriction enzyme-digested genomic DNA from 11 wasp strains. Each genomic DNA was digested with $A l u \mathrm{I}$, and the membrane was probed with the RT region of PSR c16. Fragment sizes are indicated for the A580, A480 and A370 subgroups from PSR. $\mathrm{N}$ vit, N. vitripennis; M. rap, M. raptor; other abbreviations as in Table 1
The grouping of elements on PSR with elements from the two species of Trichomalopsis was the only example where element relationships were not representative of taxonomic status (Fig. 1). In this and the previous phylogenetic analysis (McAllister and Werren 1997), the relationships among elements are very consistent with taxonomic boundaries, indicating that NATE has been transmitted vertically during the divergence of the tribe Pteromalini.

\section{Detection of elements in genomic DNA through Southern hybridization}

To confirm that elements were present in genomic DNA and to detect the presence of restriction-site defined subgroups, a Southern blot analysis was performed. Probe selection and stringency requirements were designed to concentrate the analysis on elements closely related to those on PSR. The probe consisted of the region analyzed in the sequence analysis and was derived from PSR c16. Genomic DNA was digested with $A l u \mathrm{I}$, because this restriction enzyme exhibited phylogenetically informative restriction patterns based on the sequence data.

All genomic DNAs exhibited at least faint hybridization to the probe (Fig. 2). Strong hybridization was observed to genomic DNA from the MI strain of N. vitripennis when the PSR chromosome was present, but only faint hybridization was observed in the absence of PSR (Fig. 2). A European strain (LabII) of N. vitripennis that was not carrying PSR exhibited similarly faint hybridization to the probe. Strong hybridization dependent on the presence of PSR confirms that elements are restricted to this chromosome and are not present on the A chromosomes of $N$. vitripennis.

By examining $A l u \mathrm{I}$ restriction fragments that hybridized to the probe, the presence of PSR-like elements could be visualized. Three different AluI variants were identified among the element sequences on the PSR chromosome. These element subgroups are referred to as A580, A480, and A370 (Fig. 1), and each is expected to produce a major hybridizing band of 580, 480, and $370 \mathrm{bp}$, respectively, upon digestion with $A l u \mathrm{I}$. The A580 and A480 subgroups were only identified among element sequences isolated from PSR, whereas the A370 subgroup was present among PSR, T. americanus and $T$. dubius elements (Fig. 1). Based on the hybridization results, it appears that only elements producing a $370 \mathrm{bp}$ fragment are present in the genome of $T$. americanus (Fig. 2). Strong hybridization was also observed to the $370 \mathrm{bp} A l u \mathrm{I}$ fragment in T. dubius; however, additional bands were also present in genomic DNA from this species (Fig. 2). Hybridization was detected at bands that apparently correspond to the 580 and 480 bp restriction fragments, although hybridization intensity was lower than that at the $370 \mathrm{bp}$ restriction fragment. Hybridization to the 580,480 , or $370 \mathrm{bp}$ fragments was not apparent in $N$. vitripennis, $N$. longicornis, or $N$. giraulti.

The sequence analysis revealed that elements on PSR are primarily grouped into a single clade and most closely related to elements in T. americanus. However, hybridization (Fig. 2) indicates that elements in the A580 and A480 subgroups (Fig. 1) are present in the genome of $T$. dubius. This finding is important for the relationship among the PSR elements, because it indicates these 
elements do not truly form a monophyletic group descended from a single element on PSR. In other words, multiple elements could have been present on the PSR progenitor fragment prior to its transfer from Trichomalopsis into $N$. vitripennis. An alternative possibility is that A370 elements in the T. dubius genome have lost either of two AluI restriction sites, thus yielding elements with 580 and 480 bp restriction fragments. These may not be more similar to the PSR elements in overall sequence, but may only share the lost $A l u \mathrm{I}$ restriction sites. Based on the intensity of the hybridization, however, many elements in the T. dubius genome must have lost these sites.

Strong hybridization was observed to genomic DNA from $N$. longicornis and $N$. giraulti; however, the hybridizing restriction fragments at approximately $310 \mathrm{bp}$ (Fig. 2) apparently correspond to sequences of subfamily II elements that were distantly related to those on PSR (Fig. 1). The subfamily I elements sequenced from $N$. longicornis and $N$. giraulti are approximately $88 \%$ and $84 \%$ identical, respectively, to the probe (PSR c16) sequence (Table 1). The outgroup elements (subfamily II) in $N$. longicornis/N. giraulti are about $85 \%$ identical (Table 1). Because such strong hybridization was observed to genomic DNA from $N$. longicornis and $N$. giraulti, given about a $13 \%$ sequence difference between the probe and hybridizing elements, this suggests that NATE is very abundant in these two species. Faint hybridization was observed to genomic DNA from the three species of Muscidifurax (Fig. 2), which is consistent with a relatively low copy number in these species, given the observed similarity (91\%) to the probe sequence (Table 1 ). The probe hybridized relatively strongly to $U$. rufipes (Fig. 2), and these elements are also about $91 \%$ similar to the probe (Table 1 ).

\section{Structure of PSR in relation to the A chromosomes}

Additional regions were studied to investigate the origin and structure of PSR. For this purpose, the structure of a linear region on the PSR chromosome, containing a copy of NATE (PSR c17) and undefined sequences flanking the element, was examined. An approximately $18 \mathrm{~kb}$ region of PSR was present as an insert in $\lambda$ P17. About $7 \mathrm{~kb}$ of one end of the insert is comprised of NATE, and the cloning site at this end falls within the element, causing its truncation. The structure of the DNA flanking the intact end of NATE was investigated. Plasmid subclones were obtained throughout the insert of $\lambda \mathrm{P} 17$. A restriction map of the intact NATE end ( $3^{\prime}$ or right) and the region flanking the element is presented (Fig. 3A).

Except for the copy of NATE, the content of the $\lambda \mathrm{P} 17$ region was previously unknown. No cross-hybridization was detected when the region was probed with tandemly repeated sequences that have been previously described on the PSR chromosome (Eickbush et al. 1992; McAllister 1995). Four nonoverlapping subclones were obtained that represented most of the flanking region. Using these four subclones as probes, Southern blots were performed to determine the organization of cross-hybrid- izing sequences on PSR and the A chromosomes of $N$. vitripennis, $N$. longicornis, T. americanus, and T. dubius.

Southern blots with the probes from the region flanking NATE revealed that most of this region comprises repetitive sequences. Three of the four probes cross-hybridized to repetitive sequences in genomic DNA from $N$. vitripennis, $N$. longicornis, T. americanus, and T. dubius. Subclone p17-1 hybridizes to a $2.1 \mathrm{~kb}$ HindIII fragment from PSR corresponding to the fragment from which it was derived (Fig. 3B). Hybridization at a similar-sized band was not observed in any of the other genomic DNAs. Comparisons between HindIII-digested DNA from $N$. vitripennis with and without PSR reveal the presence of other cross-hybridizing sequences on PSR (Fig. 3B). Cross-hybridizing sequences are also present in the A complement of $N$. vitripennis; however, most of the hybridization is concentrated at an approximately 800 bp HindIII fragment. This 800 bp HindIII fragment is also present in $N$. longicornis, T. america$n u s$, and $T$. dubius. In addition to hybridization at this 800 bp fragment, many larger restriction fragments hybridized to p17-1. The two Trichomalopsis species exhibited much stronger hybridization than the two Nasonia species and PSR, providing additional evidence for similarity between PSR and the genomes of the two Trichomalopsis species.

The p17-2 subclone is separated from $\mathrm{p} 17-1$ by about $300 \mathrm{bp}$ and it apparently hybridizes to the same $800 \mathrm{bp}$ HindIII fragment as p17-1, but also hybridizes to additional regions (Fig. 3C). Again, this 800 bp fragment is shared by the two Nasonia and two Trichomalopsis species. A HindIII restriction site is present in p17-2, so two adjacent HindIII fragments of 2.1 and $3.1 \mathrm{~kb}$ hybridize to this probe on PSR (Fig. 3C). Other than these two fragments, very similar hybridization patterns were exhibited in the presence or absence of PSR with the $N$. vitripennis A complement. This indicates that few or no other cross-hybridizing sequences are present on PSR. The p17-2 probe hybridized very strongly to genomic DNA from both Nasonia and Trichomalopsis species.

The p17-3 subclone, further across this region, appears to be single copy on PSR. A single $3.4 \mathrm{~kb}$ HindIII fragment hybridizes to the probe in the presence of the PSR chromosome (Fig. 3D). Cross-hybridization was not detected in genomic DNA from $N$. vitripennis, $N$. longicornis, T. americanus, and T. dubius. The image in Fig. 3D resulted from exposure for 2 days, and following exposure for 9 days no signal was detected in the other genomic DNAs. Two different methods were used in attempts to isolate a region in the A complement that is homologous to p17-3. Genomic libraries from $N$. longicornis and $N$. vitripennis (with PSR) were screened with p17-3, and no clones representing homologs were isolated. From partial sequence obtained from this region, four different primers were synthesized. These primers were used in four different combinations at medium and low stringency reaction conditions in attempts to amplify the region from $N$. vitripennis, $N$. longicornis, $N$. giraulti, and T. dubius. Although the target sequence was always amplified in the presence of PSR, neither a product of similar size nor one that cross-hybridized 


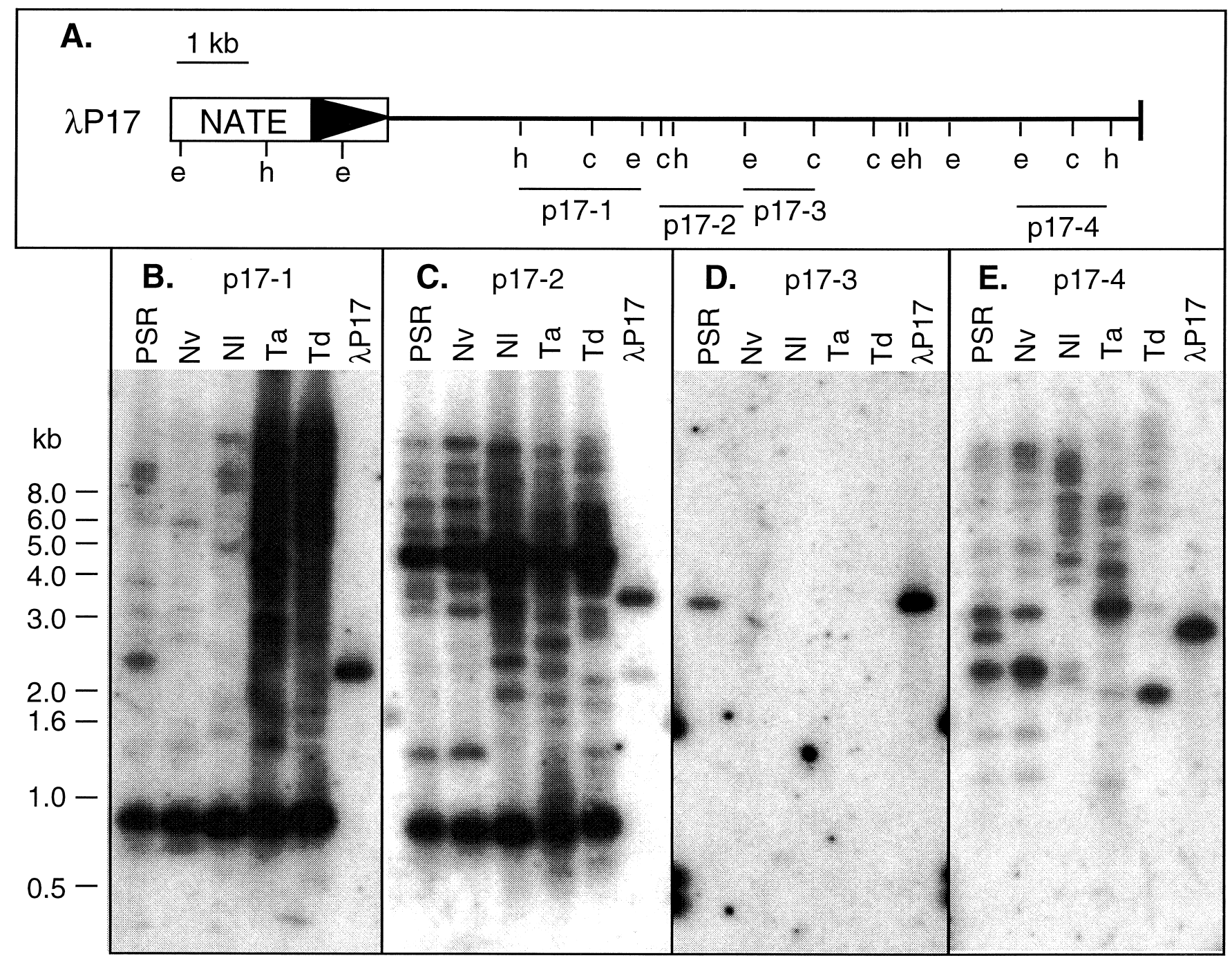

Fig. 3A-E. Organization of a cloned region of PSR and hybridization of subclones to genomic DNAs. A Restriction map and location of subclones used in the hybridization analysis. Restriction enzymes are: $c$ HincII, e EcoRI, $h$ HindIII. B-E Autoradiograms following hybridization of each subclone to genomic DNAs and

with the target sequence was amplified from the four genomic DNAs. So far, a region from an A chromosome that is homologous to p17-3 has not been isolated; thus the origin of this single-copy fragment remains elusive.

Another repetitive sequence is located in the most terminal section of this region. The subclone p17-4 was derived from a $2.7 \mathrm{~kb}$ HindIII fragment on PSR and this is visualized in the hybridization (Fig. 3E). There apparently are few or no additional cross-hybridizing sequences on PSR, because, other than the $2.7 \mathrm{~kb}$ fragment, very similar hybridization patterns were observed in the presence or absence of PSR with the $N$. vitripennis A complement. Cross-hybridizing sequences were detected in genomic DNA from all four of the wasp species, although each species exhibited a unique hybridization pattern (Fig. 3E).

Throughout this entire region, there is no detectable restriction site conservation between PSR and the crosshybridizing regions in the Nasonia or Trichomalopsis the $\lambda \mathrm{P} 17$ clone. Each of the panels contains HindIII-digested DNA from $N$. vitripennis with PSR $(P S R), N$. vitripennis without PSR $(N v), N$. longicornis $(N l), T$. americanus $(T a), T$. dubius $(T d)$, and a $\lambda$ clone containing this region of PSR $(\lambda P 17)$

genomes. The content of this region appears to be a mixture of different repetitive sequences, except for the small single-copy region (Fig. 3D). In this $18 \mathrm{~kb}$ region on the PSR chromosome, there is a copy of NATE and at least two additional repetitive elements that appear to be dispersed in the genomes of these wasp species. At least one repetitive element is present in the sequence encompassed by $\mathrm{p} 17-1$ and $\mathrm{p} 17-2$ and a different element in the region of p17-4 (Fig. 3A-C, E).

A genetic database search using sequence from these apparently repetitive sequences identified similarity to known repetitive elements. Similarity to a group of LTR (long terminal repeat) retrotransposons was revealed in three different stretches of amino acids inferred from the partial nucleotide sequences from the p17-4 subclone and adjacent subclones. The 412 retrotransposon received the top score $\left(P=7.5 \times 10^{-18}\right)$ in the BLAST search, and two other elements (osvaldo and TED) in the same retrotransposon group were included in the top five 
scores. This group of retrotransposons is very distantly related to NATE (McAllister 1995). Putative nucleotide identity between the $\mathrm{p} 17-1 / \mathrm{p} 17-2$ region and different tRNAs was identified. Although the significance of the similarity was relatively low, the repetitive sequence in this region could potentially represent a SINE-like element, since these elements have tRNA homology (Deininger 1989).

\section{Discussion}

It was previously hypothesized that the PSR chromosome recently originated in $N$. vitripennis following an interspecific hybridization with $N$. longicornis (Nur et al. 1988; Werren 1991; McAllister 1995). The PSR chromosome has a very limited distribution, and other evidence indicated that a centromeric fragment of the $N$. longicornis genome could have been transferred into $N$. vitripennis following a natural hybridization. This analysis was performed to determine whether sequences on the PSR chromosome supported the theory by sharing a high degree of similarity with homologous sequences in the $N$. longicornis genome. Comparison of the DNA content of B chromosomes with standard genomes is generally the method used for assessing the evolutionary history of Bs. Often these comparisons are difficult to interpret, because the sequences used in the analyses are repetitive and exhibit complex evolutionary patterns. A thorough analysis is required to determine the pattern exhibited by the marker before it can be used to infer the history of the B chromosome. By examining the phylogenetic relationships among the many copies of NATE isolated from the PSR chromosome and from the genomes of these parasitoid wasps, the evolutionary history of PSR has been further elucidated. The evolutionary pattern exhibited by NATE provides clear evidence that the underlying DNA that constitutes PSR was recently transferred into $N$. vitripennis. Contrary to previous predictions, however, the donor is apparently a member of the wasp genus Trichomalopsis.

For phylogenetic reconstruction, it would be preferable to compare a single-copy sequence on PSR and the homologous region from potential donor species. Such a marker has yet to be identified in the regions cloned from PSR. Although using NATE as a phylogenetic marker was not ideal, potential problems have been addressed in the analysis. One problem with the use of $N A T E$ is that multicopy elements often exhibit complex evolutionary patterns. Problems arise because the complement of elements in the genomes of a group of species may be comprised of paralogous elements that diverged prior to speciation events and orthologous elements that diverged because of speciation events (Cummings 1994). In other words, only the element lineages that diverged because of speciation events truly reflect phylogenetic history. Ancient duplications prior to speciation events and loss of element lineages can obscure evolutionary history. McAllister and Werren (1997) sequenced and analyzed 43 copies of NATE to determine its evolutionary pattern in this group of parasitic wasps.
Both orthologous and paralogous lineages were evident among these elements. However, assays for isolating elements from genomic DNAs were designed to concentrate on elements that were similar to those isolated from PSR, so that the lineage of elements encompassing those from PSR was thoroughly represented.

The phylogenetic relationships among NATE elements indicate that all those isolated from the PSR chromosome form a closely related group. Because these elements belong to the same monophyletic group as elements isolated from the two species of Trichomalopsis, the elements on PSR are apparently derived from an ancestral element or elements in the genome of a member of this genus. One possibility is that, because NATE is a retrotransposon, the elements established themselves on PSR following horizontal transfer of a single element. However, this explanation has a few unlikely requirements. First, sequence evolution of the elements on PSR must be greatly accelerated. A large number of nucleotide substitutions have occurred in the RT region among the elements on PSR, with preferential accumulation at synonymous sites (McAllister 1995). Although errorprone replication during retrotransposition may increase the substitution rate (Preston et al. 1988; Roberts et al. 1988; Li and Loeb 1992), a large number of replications would be required to generate the amount of sequence diversity observed among the elements on PSR, but there are only about nine copies of NATE on PSR (McAllister et al., in preparation). In addition to sequence divergence among elements in this internal region, sequence differences between the two LTR sequences from a single element (PSR c16) indicate that this element inserted into its present location approximately 120,000 years ago (McAllister 1995). The time required to generate the observed sequence diversity among and within elements on PSR following horizontal transfer of a single element is inconsistent with the limited geographic distribution of this chromosome. Second, the founder element must have transferred to a male $N$. vitripennis that carried PSR, and inserted into the PSR chromosome without inserting elsewhere in the genome. This is very unlikely, because in the limited region where PSR occurs, only about $5 \%$ of males carry PSR (Skinner 1983; Werren 1991; Beukeboom 1992) and this chromosome only constitutes about $5.7 \%$ of the total genome (Nur et al. 1988; Reed 1993). Horizontal transfer of a single element and establishment of this element on PSR is a convoluted explanation of the pattern exhibited by NATE.

The most plausible explanation for the relationship between copies of NATE on PSR and in the genomes of the two Trichomalopsis species is that the chromosomal fragment that constitutes PSR was transferred from a species of Trichomalopsis into N. vitripennis. Presence of multiple elements on a chromosomal fragment that was transferred into $N$. vitripennis accounts for the observed divergence among the elements on PSR. Support for this conclusion is also provided by the hybridization data (Fig. 2). Although the PSR element sequences are most closely related to sequences isolated from $T$. americanus, the hybridization data suggest the presence of undetected elements in the T. dubius genome that are more 
similar to the PSR elements. Among the sequenced elements, five of the seven elements on the PSR chromosome form a closely related group and one character that is shared by this group is a base substitution causing the loss of an $A l u \mathrm{I}$ restriction site. This subgroup was referred to as the A580 subgroup, because of the $580 \mathrm{bp}$ AluI restriction fragment in the RT region. Faint hybridization was observed to a similar-sized restriction fragment in genomic DNA from T. dubius. Presence of this restriction fragment in $T$. dubius may represent elements that were undetected when obtaining sequences. Hybridization to the $370 \mathrm{bp}$ fragment, indicative of the elements that were amplified and sequenced, was more intense than hybridization to the $580 \mathrm{bp}$ fragment; thus these elements would preferentially amplify by PCR. This interpretation indicates that elements contained in the A580 subgroup are present in the T. dubius genome, and these elements are not specific to the PSR chromosome. The same pattern was also exhibited for the A480 subgroup. However, these elements from T. dubius or other species of Trichomalopsis have not yet been isolated and sequenced.

Although multiple copies of NATE may have been present on the chromosomal fragment that was transferred from Trichomalopsis into N. vitripennis, some of the current elements on PSR may be derived through replication of elements on this chromosome. It is not known whether there are active elements on PSR. At the very least, the elements on PSR appear to be derived from a recently active lineage, because some pairs have extremely high similarity and an excess of synonymous over nonsynonymous substitutions (McAllister 1995). If active elements are present, however, the inheritance pattern of PSR prevents transfer of elements to the A chromosomes in the general population of $N$. vitripennis. The A complement of each male that carries PSR is destroyed after fertilization, owing to PSR action. Therefore, the standard genome of $N$. vitripennis that has been a potential recipient of elements from PSR was extinguished following exposure to this chromosome. This means that transposon flow between PSR and the standard $N$. vitripennis genome is unidirectional-PSR can potentially acquire elements from A chromosomes, but the reverse is prevented. Three iso-female lines of $N$. vitripennis obtained from the geographic region where PSR occurs were screened by Southern hybridization for the presence of PSR-like elements (B. McAllister, unpublished). No PSR-like elements were detected in the $N$. vitripennis lines, supporting the notion that the transmission mechanism of PSR prevents the movement of elements from PSR to the A chromosomes.

The best explanation for the transfer of a chromosomal fragment from a species of Trichomalopsis to N. vitripennis is through copulation and fertilization. This requires two factors: ecological association of the two species, and breakdown in pre-zygotic isolating mechanisms. The genus Trichomalopsis (previously Eupteromalus in North America) contains many species and is distributed in both Nearctic and Palearctic regions (Burks 1979; Boucek and Rasplus 1991). Strains of $T$. dubius and T. americanus used here were not collected from the geographic region where PSR is present in $N$. vitripennis. The T. americanus strain was isolated as a parasite on muscoid flies (M. domestica and Stomoxys calcitrans) occurring in a feedlot near Lincoln, Nebraska, USA (J. Petersen, pers. commun.) and T. dubius was isolated from a dairy near Ithaca, New York, USA (Hoebeke and Rutz 1988). There are no records of either species being collected from the Great Basin region of North America, although the distribution of T. americanus includes the northwestern United States (Burks 1979). During censuses of natural populations of Nasonia, however, wasps tentatively identified as $T$. dubius were collected in the region where PSR is found (J. Werren, unpublished data). In the Great Basin region, $T . d u b$ ius was occasionally collected from baits placed near carcasses and in feedlots, the primary habitats for $N$. vitripennis. Other than these limited observations on distributions and habitats, indicating a slight but not overwhelming ecological association, there is little known about potential contact between $N$. vitripennis and species of Trichomalopsis. In the event that a species of Trichomalopsis was in contact with $N$. vitripennis, copulation and fertilization were required to transfer the chromosomal fragment. In the laboratory, attempts at crossing either T. dubius or T. americanus with $N$. vitripennis have been unsuccessful. Males of either genus would court females of the other; however, females were not receptive (B. McAllister, unpublished). This suggests that strong pre-zygotic isolating mechanisms are present, so failure of these isolating mechanisms must be rare.

Although hybridization between a Trichomalopsis species and $N$. vitripennis may have been an extremely rare event, the phylogenetic pattern of NATE is strong evidence for hybridization and transfer of a chromosomal fragment that constitutes PSR into $N$. vitripennis. One major issue remains unresolved from the analysis of NATE. Was the chromosomal fragment a component of the normal chromosomal complement of the donor species prior to transfer, or did the male wasp that hybridized with $N$. vitripennis carry a fully functional PSR chromosome? The first scenario will be referred to as the Hybrid Generation model. Under this model, hybridization resulted in the fragmentation of the paternal (Trichomalopsis) genome; a haploid (male) zygote containing the maternal ( $N$. vitripennis) genome plus the fragment developed, and the fragment eventually evolved into the extant PSR chromosome. Generation of centromeric fragments during failed development is not unprecedented in Nasonia (Ryan et al. 1985). The evolution of PSR from a chromosomal fragment requires rapid evolution of the ability to destroy the paternal genome, because if the haploid supernumerary was transmitted to females it would suffer substantial loss during meiosis (Ryan et al. 1985; Nur et al. 1988; Beukeboom and Werren 1993b). Hybrid Transfer of PSR is the alternative model for originating PSR in $N$. vitripennis. According to this model, PSR is not unique to $N$. vitripennis, but the $\mathrm{B}$ chromosome is also present in at least one species of Trichomalopsis. A male wasp of this Trichomalopsis species carried PSR and hybridized with a $N$. vitripennis female, thus transferring the chromo- 
some. The action of PSR (destruction of the paternal genome) would have eliminated the effects of hybrid inviability.

Verification of either of the above models will be difficult, but the microstructural comparison between PSR and the genomes of the Nasonia and Trichomalopsis species provides an initial assessment of the Hybrid Generation hypothesis. Examination of a linear region of PSR in comparison with genomic DNA from these species indicated that a similar region was not present in the A chromosomes. Under the Hybrid Generation model, PSR was recently derived from a genomic region in a donor species. Similarity should be relatively high between PSR and the progenitor region. To account for the lack of similarity between PSR and the A chromosomes of these wasps, either the donor species has yet to be identified or rapid structural evolution has occurred on PSR and/or the region where PSR was derived. Both of these requirements are entirely plausible.

The microstructural analysis indicated that the linear region of PSR contained an abundance of repetitive sequences. All regions of the chromosome thus far examined contain dispersed or tandemly repeated sequences, except for the small single-copy region identified in this study. Furthermore, PSR has several sequences that are entirely unique. No cross-hybridization has been detected between the single-copy region of PSR and the chromosomes from the Trichomalopsis and Nasonia species. Also, three families of tandemly repeated sequences on PSR have not been identified in standard genomes of the Nasonia or Trichomalopsis species (Eickbush et al. 1992; McAllister, unpublished). Based on the molecular organization of PSR, the chromosome may be in the later stages of molecular degeneration, characterized by the accumulation of repetitive elements, deletions, and duplications (Amos and Dover 1981; Green 1990; Steinemann and Steinemann 1992). Evidence for a degenerate PSR chromosome could favor the Hybrid Transfer model, suggesting PSR is not recently derived from a standard chromosomal fragment, but an old B chromosome that recently transferred species. This model is also logistically more simple, because PSR would have presumably possessed the ability to maintain itself as a B chromosome. The Hybrid Generation model requires drive to evolve.

Under either model, PSR had to originate at some point. The difference is whether the de novo origin of PSR occurred at the same time as transfer into $N$. vitripennis, or whether de novo origin predates this event. Whichever hypothesis is correct, further analysis of this problem will provide insight into the process of molecular degeneration. Although the phenomenon of molecular degeneration is commonly exhibited by B chromosomes and sex-limited chromosomes, the population genetic forces underlying the process remain unknown (Rice 1987; Charlesworth 1991, 1996). Theory and an artificial simulation of Y-chromosome evolution indicate that the absence of recombination is a major factor (Rice 1994). Additionally, studies of the neo-Y chromosome of Drosophila miranda show that transposable elements have accumulated on that degenerating chromosome
(Steinemann and Steinemann 1991, 1992). The study of molecular degeneration on B chromosomes will provide valuable insights into the process of molecular degeneration, because B and Y (or W) chromosomes are affected differently by selection. Whereas $\mathrm{Y}$ chromosomes are subject to purifying selection on genetic loci that are shared with the X (Fisher 1935), B chromosomes have no selection pressure to maintain unnecessary genetic loci. Therefore, further studies of PSR and other B chromosomes will provide useful examples for examining the effects of different population genetic forces on the process of molecular degeneration.

Acknowledgments. The authors thank T. Eickbush, U. Nur, H. Ochman, and B. Charlesworth for commenting on earlier drafts of the manuscript. Sincere gratitude is expressed to S. Long (Cornell University) and J. Petersen (University of Nebraska) for providing wasp strains. The project is part of the Ph.D. dissertation submitted by B. McAllister, who was partially supported by an Ernst Caspari Fellowship. This research was supported by a grant from the National Science Foundation to J. Werren.

\section{References}

Altschul SF, Gish W, Miller W, Myers EW, Lipman DJ (1990) Basic local alignment search tool. J Mol Biol 215:403-410

Amos A, Dover G (1981) The distribution of repetitive DNAs between regular and supernumerary chromosomes in species of Glosnia (Tsetse): a two-step process in the origin of supernumeraries. Chromosoma 81:673-690

Ausubel FM, Brent R, Kingston RE, Moore DD, Seidman JG, Smith JA, Struhl K (eds) (1992) Current protocols in molecular biology. John Wiley and Sons, New York, p 7.3.7

Beukeboom LW (1992) Genetics and population biology of the Paternal Sex Ratio (PSR) chromosome. PhD Dissertation, University of Rochester, Rochester, New York

Beukeboom LW (1994) Bewildering Bs: an impression of the 1st B-chromosome conference. Heredity 73:328-336

Beukeboom LW, Werren JH (1993a) Transmission and expression of the parasitic paternal sex ratio (PSR) chromosome. Heredity 70:437-443

Beukeboom LW, Werren JH (1993b) Deletion analysis of the selfish B chromosome, Paternal Sex Ratio (PSR), in the parasitic wasp Nasonia vitripennis. Genetics 133:637-648

Boucek Z, Rasplus JY (1991) Illustrated key to west-palearctic genera of Pteromalidae. Institut National de la Recherche Agronomique, Paris, p 140

Burks BD (1979) Family Pteromalidae. In: Krombein KV, Hurd PD, Smith DR, Burks BD (eds) Catalog of Hymenoptera in America north of Mexico. Smithsonian Institution Press, Washington, DC, pp 768-835

Cabot EL, Beckenbach AT (1989) Simultaneous editing of multiple nucleic acid and protein sequences using ESEE. Comput Appl Biosci 5:233-234

Charlesworth B (1991) The evolution of sex chromosomes. Science 251:1030-1033

Charlesworth B (1996) The evolution of chromosomal sex determination and dosage compensation. Curr Biol 6:149-162

Cummings MP (1994) Transmission patterns of eukaryotic transposable elements: arguments for and against horizontal transfer. Trends Ecol Evol 9:141-145

Deininger PL (1989) SINEs: short interspersed repeated DNA elements in higher eukaryotes. In: Berg DE, Howe MM (eds) Mobile DNA. American Society for Microbiology, Washington, DC, pp 619-636

Eickbush DG, Eickbush TH, Werren JH (1992) Molecular characterization of repetitive DNA sequences from a B chromosome. Chromosoma 101:575-583 
Fisher RA (1935) The sheltering of lethals. Am Nat 69:446-455

Gish W, States DJ (1993) Identification of protein coding regions by database similarity search. Nat Genet 3:266-272

Green DM (1990) Muller's ratchet and the evolution of supernumerary chromosomes. Genome 33:818-824

Green DM, Zeyl CW, Sharbel TF (1993) The evolution of hypervariable sex and supernumerary (B) chromosome in the relict New Zealand frog, Leiopelma hochstetteri. J Evol Biol 6:417-441

Gutknecht J, Sperlich D, Bachmann L (1995) A species specific satellite DNA family of Drosophila subsilvestris appearing predominantly in B chromosomes. Chromosoma 103:539-544

Hoebeke ER, Rutz DA (1988) Trichomalopsis dubius (Ashmead) and Dibrachys cavus (Walker): newly discovered pupal parasitoids (Hymenoptera: Pteromalidae) of house flies and stable flies associated with livestock manure. Ann Entomol Soc Am 81:493-497

Jamilena M, Ruiz Rejón C, Ruiz Rejón M (1994a) A molecular analysis of the origin of the Crepis capillaris $\mathrm{B}$ chromosome. J Cell Sci 107:703-708

Jamilena M, Garrido-Ramos M, Ruiz Rejón C, Ruiz Rejón M (1994b) Molecular relationships between the A and B chromosomes of Crepis capillaris. Heredity 73:527-531

Jamilena M, Garrido-Ramos M, Ruiz Rejón M, Ruiz Rejón C, Parker JS (1995) Characterization of repeated sequences from microdissected B chromosomes of Crepis capillaris. Chromosoma 104:113-120

John UP, Leach CR, Timmis JN (1991) A sequence specific to B chromosomes of Brachycome dichromosomatica. Genome 34:739-744

Jones RN (1991) B-chromosome drive. Am Nat 137:430-442

Jones RN, Rees H (1982) B chromosomes. Academic Press, New York

Leach CR, Donald TM, Franks TK, Spiniello SS, Hanrahan CF, Timmis JN (1995) Organisation and origin of a B chromosome centromeric sequence from Brachycome dichromosomatica. Chromosoma 103:708-714

Li J, Loeb LA (1992) Fidelity of HIV-1 reverse transcriptase copying RNA in vitro. Biochemistry 31:954-958

McAllister BF (1995) Isolation and characterization of a retroelement from a B chromosome (PSR) in the parasitic wasp Nasonia vitripennis. Insect Mol Biol 4:253-262

McAllister BF, Werren JH (1997) Phylogenetic analysis of a retrotransposon with implications for strong evolutionary constraints on reverse transcriptase. Mol Biol Evol 14:69-80

McVean GT (1995) Fractious chromosomes: hybrid disruption and the origin of selfish genetic elements. BioEssays 17:579582
Nur U, Werren JH, Eickbush DG, Burke WD, Eickbush TH (1988) A "selfish" B chromosome that enhances its transmission by eliminating the paternal genome. Science 240:512-514

Preston BD, Poiesz BJ, Loeb LA (1988) Fidelity of HIV-1 reverse transcriptase. Science 242:1168-1171

Reed KM (1993) Cytogenetic analysis of the paternal sex ratio chromosome of Nasonia vitripennis. Genome 36:157-161

Reed KM, Werren JH (1995) Induction of paternal genome loss by the paternal-sex-ratio chromosome and cytoplasmic incompatibility bacteria (Wolbachia): a comparative study of early embryonic events. Mol Reprod Dev 40:408-418

Rice WR (1987) Genetic hitchhiking and the evolution of reduced genetic activity of the Y sex chromosome. Genetics 116:161167

Rice WR (1994) Degeneration of a nonrecombining chromosome. Science 263:230-232

Roberts JD, Bebenek K, Kunkel TA (1988) The accuracy of reverse transcriptase from HIV-1. Science 242:1171-1173

Ryan SL, Saul GB II, Conner GW (1985) Aberrant segregation of $\mathrm{R}$-locus genes in male progeny from incompatible crosses in Mormoniella. J Hered 76:21-26

Sapre AB, Deshpande DS (1987) Origin of B chromosomes in Coix L. through spontaneous interspecific hybridization. J Hered 78:191-196

Schartl M, Nanda I, Schlupp I, Wilde B, Epplen JT, Schmid M, Parzefall J (1995) Incorporation of subgenomic amounts of DNA as compensation for mutational load in a gynogenetic fish. Nature 373:68-71

Skinner SW (1983) Extrachromosomal sex ratio factors in the parasitoid wasp Nasonia (=Mormoniella) vitripennis. PhD Dissertation, University of Utah, Salt Lake City, Utah

Steinemann M, Steinemann S (1991) Preferential Y chromosomal location of TRIM, a novel transposable element of Drosophila miranda, obscura group. Chromosoma 101:169-179

Steinemann M, Steinemann S (1992) Degenerating Y chromosome of Drosophila miranda: a trap for retrotransposons. Proc Natl Acad Sci USA 89:7591-7595

Swofford DL (1991) PAUP: phylogenetic analysis using parsimony, version 3.1.1. Smithsonian Institution, Washington, DC

Werren JH (1991) The paternal-sex-ratio chromosome of Nasonia. Am Nat 137:392-402

Werren JH, van den Assem J (1986) Experimental analysis of a paternally inherited extrachromosomal factor. Genetics 114:217-233

Werren JH, Nur U, Eickbush DG (1987) An extrachromosomal factor causing loss of paternal chromosomes. Nature 327:7576 